\title{
Suburbanes Wachstum von Bevölkerung und Beschäftigung als Weg zur dezentralen Mischung von Wohnen und Arbeiten? - Eine empirische
}

Untersuchung am Beispiel der Nordwestschweiz

\section{Rainer Volman, Basel}

\section{Einführung in die Thematik}

Die räumliche Mischung von Flächennutzungen für das Wohnen, Arbeiten oder die Erholung ist ein Leitbild der Raumplanung, von dem man sich unter anderem eine entlastende Wirkung auf das Verkehrsaufkommen verspricht. Durch die möglichst kompakte räumliche Mischung von Flächennutzungen soll die alltägliche Lebensführung ohne lange Wege möglich werden. Die anhaltende Tendenz zur Verlagerung des Siedlungswachstums, der Einkaufsmöglichkeiten und Freizeitfunktionen von den kompakten Agglomerationskernen in das Umland könnte jedoch auch zu einer Verschärfung der räumlichfunktionalen Trennung oder zu einer dezentralen Mischung der Flächennutzungen führen. Ziel dieser Arbeit ist es, für die Bereiche Wohnen und Arbeiten zu untersuchen, ob die Verlagerung des Wachstums ins Umland zu einer stärkeren räumlichen Mischung dieser beiden Funktionen geführt hat, oder ob sich die räumliche Trennung von Wohnen und Arbeiten verstärkt hat. Ersteres würde die Realisierungschancen von raumordnerischen Leitbildern erhöhen, die auf eine dezentrale Nutzungsmischung in der Fläche abzielen. Letzteres würde aus Sicht der Verkehrsvermeidung ein verstärktes Gegensteuern gegen die Landschaftszersiedelung anzeigen beziehungsweise für eine gezieltere Planung von Nutzungsmischungen sprechen.

\section{Nutzungsmischung als ein Ziel der Raumordnung}

Leitbilder für die räumliche Ordnung hatten im Verlauf der Zeit unterschiedliche thematische und räumliche Schwerpunkte und waren dabei immer auch ein Spiegel der gerade vorherrschenden Problemlagen. Frühe Siedlungskonzepte wie die Gartenstadtidee (HowarD 1907), aber auch noch die Thesen zur räumlichen Trennung unterschiedlicher Flächennutzungen in der Charta von Athen 1933 (vgl. HilPert 1988) oder das Leitbild der «gegliederten und aufgelockerten Stadt» (GöDERITZ et al.1957) bezogen sich auf die räumliche Organisation von Stadtregionen mit dem Ziel der Trennung des Wohnens von den emmissionsreichen Produktionsstätten des Industriezeitalters. In der Zeit nach dem Zwei- ten Weltkrieg beherrschten an den erklärenden Konzepten von CHRISTALLER (1933) ausgerichtete Leitbilder zur räumlichen Verteilung zentraler Orte die Raumplanung. Dabei stand zunächst die Versorgung von ländlichen Räumen mit öffentlicher Infrastrukur im Vordergrund. Ergänzt wurden diese entwicklungspolitisch motivierten Leitbilder durch die Ausweisung von Fördergebieten, in denen gezielt privatwirtschaftliche Aktvitiäten angeregt werden sollten (z.B. in der Schweiz das Bundesgesetz über die Investitionshilfe für Berggebiete). Auch die Ausweisung von «Entwicklungsachsen» (IsBaRY 1969) ist diesen entwicklungspolitischen Konzepten zuzurechnen. Die Kombination von Zentren und Achsen zu punktachsialen Konzepten der Siedlungsentwicklung findet sich noch heute in der Landes- und Regionalplanung, beispielsweise beim Konzept des vernetzten Städtesystems Schweiz (BundeSAMT Für Raumplanung 1996: 42ff.). Seit den siebziger Jahren treten neben den entwicklungspolitischen Aspekten zunehmend ökologische Aspekte der Siedlungsentwicklung in den Vordergrund. Dies gilt besonders für die unter dem Schwund von Freiflächen und zunehmender Verkehrsbelastung leidenden Agglomerationen.

Das Konzept der dezentralen Konzentration, bei dem Orte im Umland von Ballungszentren gezielt ausgebaut werden, erfüllt als regionales Leitbild eher eine ordnende Aufgabe: Durch die Konzentration der Wachstumsdynamik der Agglomerationen auf wenige Zentren und Subzentren sollen noch bestehende Freiräume vor Zersiedelung bewahrt, Infrastrukturkosten gespart und der Verkehr auf wenigen Achsen gebündelt werden (z.B. BUNDESAMT FüR Raumplanung 1996: 44; Apel, Henckel et al. 1995: 46f.). Die Bündelung von Aktivitäten in wenigen Zentren soll durch die wegesparende Zuordnung von Standorten des Wohnens, Arbeitens oder Einkaufens auch der Verkehrsvermeidung dienen: Je enger diese Standorte zusammenrücken, desto geringer werden die Wegstrecken, die für die alltägliche Lebensführung notwendig sind (WürdEMANN 1998: 356, Apel, Henckel et al. 1995: 49ff., Kutter \& Stein 1996, KNOFLACHER 1994).

Entgegen dem Leitbild der dezentralen Konzentration zeigen in Deutschland und der Schweiz gerade die Gemeinden mit zentralörtlichen Funktionen einen unterdurchschnittlichen Zuwachs der Bevölkerungszahl bzw. überdurchschnittliche Verluste bei den Beschäftigtenzahlen (SCHUler et al. 
1997: 34, 154, Hesse \& Schmitz 1998: 436, vgl. Kap. 4.1). Angesichts dieser mangelnden Umsetzung des Leitbilds der dezentralen Konzentration werden in jüngster Zeit Vorschläge für eine Orientierung an dem möglicherweise realitätsnäheren Ziel einer dezentralen «Nutzungsmischung in der Fläche» (z.B. innerhalb von Gemeinden oder auf Ebene von Gruppen benachbarter Gemeinden) laut (SIEvERTS 1997, WüRDEMANN 1998: 367) (vgl. Abb. 1 a versus b, c). Die Überlegung ist, dass auch dabei Siedlungsmuster entstehen können, die kurze Wege zwischen Wohnen, Arbeiten usw. möglich machen: Wenn sich die Zersiedelung nicht in grossräumig getrennten, monofunktionalen Teilräumen vollzieht, sondern sich auch im Umland der Agglomerationskerne ein kleinräumig durchmischtes Muster von Wohn-, Arbeits-, Einkaufs- und Freizeitstätten herausbildet, dann würden die immer grösseren Ausdehnungen der Agglomerationen als Ganzes nicht unbedingt zu immer grösseren Wegestrecken der einzelnen Einwohner führen. Diese Art der Siedlungsmuster wird im folgenden als «dezentrale Nutzungsmischung in der Fläche» bezeichnet. Denkbar wäre die Mischung innerhalb von Gemeinden oder zumindest im Kreis benachbarter Gemeinden wie z.B. Bezirken (Abb. $1 \mathrm{~b}$ und $\mathrm{c}$ ).

\section{Ziel und Methodik der Untersuchung}

\subsection{Ziel der Untersuchung}

Die geschichtliche Vielfalt raumordnerischer Leitbilder zeigt, dass die Beurteilung der Siedlungsentwicklung unter verschiedensten Blickwinkeln erfolgen kann. Beispiele sind die Versorgung der Bevölkerung mit öffentlicher Infrastruktur, die Vermeidung der Nachbarschaft störender Flächennutzungen, die räumliche Verteilung der Chancen für die wirtschaftliche Entfaltung oder die Schonung von Freiflächen.

Die folgende Untersuchung bezieht sich auf die Leitbilder zur «dezentralen Nutzungsmischung in der Fläche». Die Siedlungsentwicklung wird gemäss deren Intention unter dem Gesichtspunkt der räumlichen Funktionsmischung, die eine Lebensführung der kurzen Wege ermöglichen soll, untersucht. Für den Untersuchungsraum Nordwestschweiz soll geklärt werden, inwieweit das gegenwärtigen Bevölkerungs- und Arbeitsplatzwachstum im Umland des Agglomerationskerns Basel tatsächlich per Saldo mit einer räumlichen Mischung von Wohnen und Arbeiten einhergeht und falls ja, ob diese Mischung auf Gemeinde- oder erst auf der Ebene von Bezirken zu beobachten ist. Sollte das Bevölkerungs- und Beschäftigungswachstum im Um-

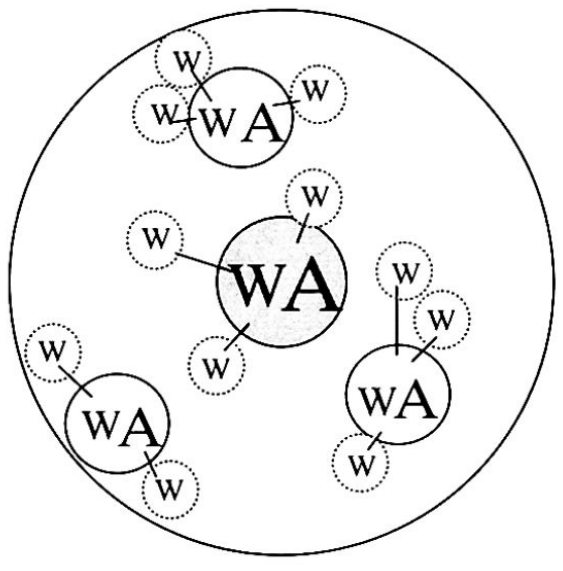

a: Dezentrale Konzentration von Wohnen und Arbeiten

$\mathrm{W}=$ Standorte Wohnen $\mathrm{A}=$ Standorte Arbeiten
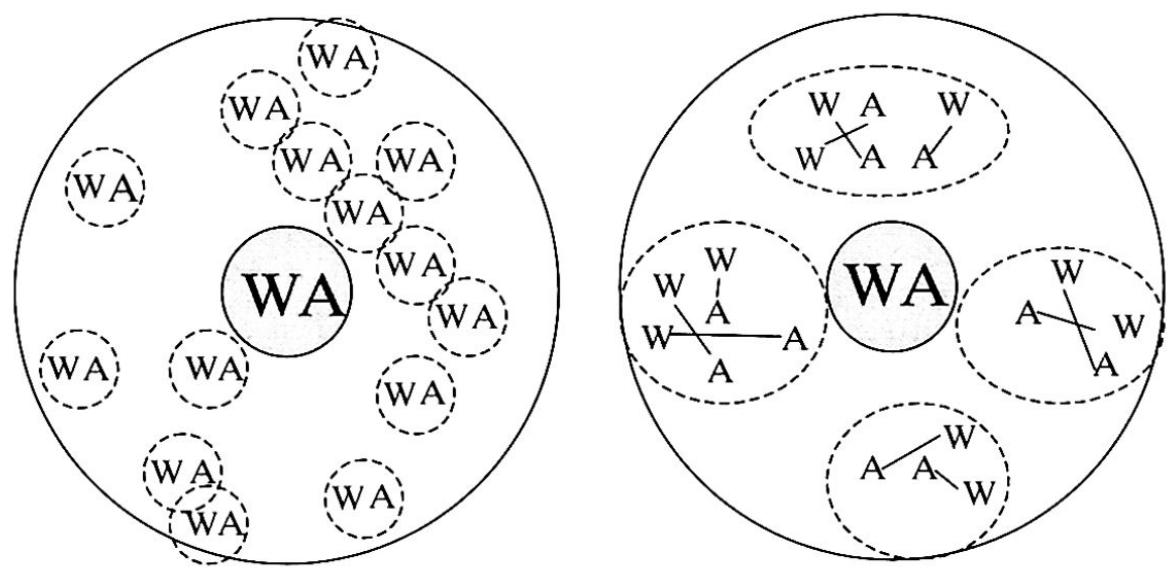

«Dezentrale Mischung von Wohnen und Arbeiten in der Fläche» b: Innerhalb der Gemeinden

c: Auf der Ebene von Bezirken

Abb. 1: Leitbilder der räumlichen Mischung von Wohnen und Arbeiten

Planning concepts pertinent to the distribution of people and jobs

Modèles de la combinaison spatiale de l'habitat et du travail

Quelle: eigener Entwurf 
land (z.B. innerhalb von Gemeinden oder innerhalb von Bezirken) tatsächlich zu einer räumlich ausgewogeneren Mischung von Wohnen und Arbeiten führen, würde dies die Vorstellungen zur dezentralen Nutzungsmischung in der Fläche als realistischeres Alternativmodell zur dezentralen Konzentration stärken: Statt einen aussichtslos erscheinenden Kampf für die Ballung der Siedlungsentwicklung in wenigen Zentren und Subzentren zu führen, könnte die Raumplanung dann eher die bereits laufenden Vorgänge der dezentralen, lokalen Nutzungsmischung begleiten. Gehen die aktuellen Vorgänge dagegen mit einer weiteren Trennung von Wohnen und Arbeiten einher, würde dies bedeuten, dass die Zersiedelung nicht «automatisch» zur dezentralen Nutzungsmischung führt. Auch die dezentrale Nutzungsmischung würde dann nicht ohne gezieltes Eingreifen der Raumplanung verwirklicht werden können.

Untersucht wird die räumliche Mischung von Wohnen und Arbeiten. Die Standorte von Wohn- und Arbeitsstätten sind von besonderem Interesse für die Verkehrsentstehung, da sie die Wegemuster noch eher prägen als beispielsweise Standorte von Freizeiteinrichtungen, die nur unregelmässig besucht werden und bei denen auch von einem Haushalt ein breites, ständig wechselndes Spektrum von Standorten frequentiert wird (WÜRDEMANN 1998: 356, OPASCHOWSKI 1999).

\subsection{Untersuchungsmethodik}

Gemäss dem Untersuchungsziel wird die räumliche Mischung von Wohnen und Arbeiten unter dem Gesichtspunkt der Verkehrsvermeidung ermittelt. Rein rechnerisch ist die optimal ausgewogene Mischung von Wohnen und Arbeiten in einem Gebiet dann erreicht, wenn der arbeitenden oder arbeitssuchenden Wohnbevölkerung (Erwerbspersonen) die gleiche Anzahl an Beschäftigungsmöglichkeiten (gemessen als Beschäftigte) vor Ort zur Verfügung steht. Als Mass für die Verbesserung oder Verschlechterung der Ausgewogenheit der Mischung von Wohnen und Arbeiten in einem Gebiet wird die «Standortdivergenz» (D) definiert. Sie ist der Betrag der Differenz zwischen in einem Gebiet wohnhaften Erwerbspersonen (EP) und dort arbeitenden Beschäftigten (B).

$D=|E P-B|$

Im Idealfall beträgt $\mathrm{D}=0$. Je grösser $\mathrm{D}$, desto unausgewogener sind Erwerbspersonen und Beschäftigte in einem Gebiet vertreten, weil Erwerbspersonen oder Beschäftigte fehlen. Eine geringere Anzahl von Beschäftigungsmöglichkeiten im Vergleich zu den Erwerbspersonen könnte nur durch Auspendeln der Ortsansässigen aus dem Gebiet ausgeglichen werden, eine geringere Anzahl von Erwerbspersonen im Vergleich zu den Beschäftigungsmöglichkeiten nur durch
Einpendeln von Auswärtigen. Nicht berücksichtigt werden hier möglicherweise unterschiedliche Erwartungen von Arbeitgebern und -nehmern bezüglich Arbeitszeit, Gehalt und Qualifikation der Arbeit. Solche unterschiedliche Erwartungen können dazu führen, dass trotz zahlenmässiger Gleichheit von Erwerbspersonen- und Beschäftigtenzahlen in einem Ort nicht alle örtlichen Beschäftigungsmöglichkeiten durch vor Ort wohnhafte Erwerbspersonen besetzt werden. Die Nähe von Wohn- und Arbeitsstätten allein garantiert also noch nicht, dass die Wohnungen in der Nähe von Arbeitsplätzen auch von den Beschäftigten dieser Arbeitsplätze bezogen werden (vgl. Kahnert 1998: 516). Eine geringe Standortdivergenz ist somit für ein geringes Aufkommen im überörtlichen Berufspendlerverkehr eine noch nicht hinreichende, jedoch notwendige Bedingung; denn ohne eine geringe Standortdivergenz ist ein geringes Berufspendler-Verkehrsaufkommen nicht einmal theoretisch möglich.

Die Standortdivergenz kann für unterschiedliche räumliche Bezugseinheiten berechnet werden. Je kleiner die untersuchten Raumeinheiten gewählt werden, desto schärfer sind die Anforderungen an die räumliche Nähe von Wohnen und Arbeiten, bis die ideale Nutzungsmischung mit $\mathrm{D}=0$ erreicht ist. Bei einer quartiersbezogenen Untersuchung müsste beispielsweise die passende Anzahl Beschäftigungsmöglichkeiten innerhalb desselben Quartiers vorliegen. Die Datenlage erlaubt für diese Untersuchung aber als feinste räumliche Auflösung lediglich die Gemeindeebene. Die Standortdivergenz einer Gemeinde $\mathrm{i}\left(\mathrm{D}_{\mathrm{i}}\right)$ erreicht ihren Optimalwert $\mathrm{D}_{\mathrm{i}}=0$, wenn innerhalb der Gemeinde jeder Erwerbsperson rechnerisch ein Beschäftigter gegenübersteht, unabhängig davon, in welchem Quartier dies der Fall ist.

\section{$D_{i}=\left|E P_{i}-B_{i}\right|$}

Die Standortdivergenz wird ausserdem auch für die Ebene von Bezirken ermittelt. Da die räumliche Nähe von Wohnen und Arbeiten das Hauptinteresse dieser Untersuchung bildet, wurden die Bezirksexklaven Kleinlützel, Rodersdorf, Metzerlen, Hofstetten, Witterswil oder Bättwil den jeweils am besten erreichbaren, angrenzenden Bezirken zugeschrieben, Bözen, Elfingen und Effingen dem Bezirk Laufenburg (vgl. Karte 1).

Der Optimalwert der Standortdivergenz auf Ebene eines Bezirks $j\left(D_{j}=0\right)$ wird bereits dann erreicht, wenn zumindest innerhalb der Bezirke die Anzahl der Erwerbspersonen und Beschäftigten übereinstimmt, auch wenn in einzelnen Gemeinden der Bezirke ein Mangel an Erwerbspersonen oder Beschäftigten besteht. Das heisst, fehlende Beschäftigungsmöglichkeiten oder Erwerbspersonen in einer Gemeinde 


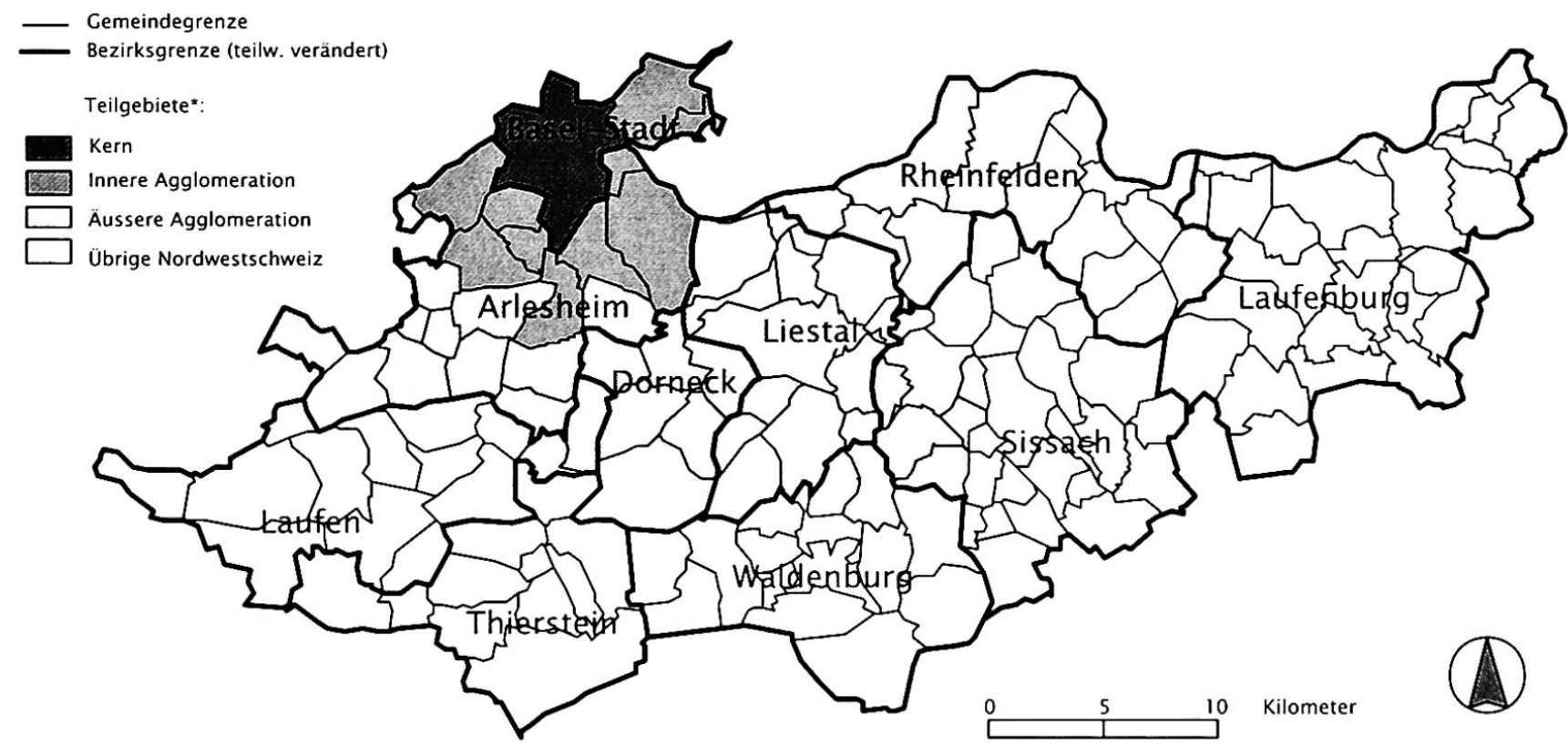

* Abgrenzung gemäss SCHULER et al. (1997): Innere Agglomerationsgemeinden bis 1950, äussere Agglomeration = Agglomerationsgemeinden ab 1950

Karte 1: Untersuchungsrelevante Teilräume der Nordwestschweiz

Regional units relevant to the area of investigation (North-West Switzerland)

Subdivision pertinentes du nord-ouest de la Suisse

Kartengrundlage: BFS GEOSTAT/L+T; Kartographie und Bearbeitung: R.VoLMAN

können mit passenden Überschüssen anderer Gemeinden des Bezirks ausgeglichen werden. Die Standortdivergenz auf Bezirksebene ist durch diesen statistischen Effekt immer kleiner oder gleich der Summe der zugehörigen gemeindlichen Standortdivergenzen.

$D_{j}=\left|E P_{j}-B_{j}\right|$

Mit dem Zeitvergleich der Standortdivergenzen auf Gemeinde- oder Bezirksebene kann gemessen werden, in welche Richtung und in welchem Ausmass sich die Nutzungsmischung auf verschiedenen räumlichen Ebenen verändert hat. Für die Untersuchung ist dabei neben der Veränderung der Standortdivergenzen in einzelnen Gemeinden oder Bezirken vor allem von Interesse, wie sich die Nutzungsmischung per Saldo in der Nordwestschweiz verändert hat. Dazu werden die gemeinde- oder die bezirksbezogenen Standortdivergenzen aufsummiert.

Datengrundlage zur Berechnung der Standortdivergenzen waren am Arbeitsort erhobene Beschäftigtenzahlen aus den Betriebszählungen 1991 und 1998 sowie Bevölkerungszahlen aus den laufenden Fortschreibungen der Volkszählung (Statistik des jährlichen Bevölkerungsstandes: ESPOP). Da Erwerbspersonenzahlen nur für die Jahre der Volkszählung zur Verfügung stehen, wurden die Bevölkerungszahlen 1998 mit der Erwerbsquote aus dem Volkszählungsjahr
1990 multipliziert, um einen Näherungswert für die Erwerbspersonenzahlen $1998 \mathrm{zu}$ erhalten $\left(\mathrm{EP}_{1998}=\right.$ $\left.\mathrm{EW}_{1098} * \mathrm{EP}_{1990} / \mathrm{EW}_{1090}\right)$. Berücksichtigt werden damit nur Veränderungen der Erwerbspersonenzahl in einer Gemeinde, die auf Veränderungen der örtlichen Bevölkerungszahl basieren, nicht aber solche, die aus lokalen Veränderungen der Erwerbspersonenquote entstanden.

Der Untersuchungszeitraum von 1991 bis zur jüngsten Betriebszählung 1998 umfasst eine Phase des konjunkturell bedingten Abbaus der Beschäftigtenzahlen in der Nordwestschweiz. Die Veränderung der Beschäftigtenzahlen war damit insgesamt im Verhältnis zu den Bevölkerungs- und Erwerbspersonenzahlen ungünstiger, als dies langfristig zu erwarten ist. Dieser konjunkturelle Effekt beeinflusst auch die örtlichen Veränderungen der Standortdivergenz: Die kurzfristig relativ ungünstige Entwicklung der Beschäftigtenzahlen führt dazu, dass die Standortdivergenz in Gemeinden mit anfänglichem Mangel an Beschäftigten überschätzt, in Gemeinden mit anfänglichem Überschuss an Beschäftigten unterschätzt wird. Um diese kurzfristigen, konjunkturbedingten Effekte auszublenden, wurde neben der tatsächlichen Änderung der Standortdivergenz auch eine konjunkturbereinigte Veränderung der Standortdivergenz $\left(D_{i}^{*}\right)$ berechnet. Diese beinhaltet lediglich den Einfluss der räumlichen Verlagerung der 
Schwerpunkte von Wohnen und Arbeiten, hält die Summe der Erwerbspersonen und Beschäftigten in der Nordwestschweiz aber fiktiv konstant. Die gerade relativ ungünstige Entwicklung der Beschäftigten- im Verhältnis zu den Erwerbspersonenzahlen wird damit ausgeblendet. Um alleine die neue räumliche Verteilung der Erwerbspersonen und Beschäftigten im Jahr 1998 zu berücksichtigen, werden die neuen Anteile der Gemeinden an den Erwerbspersonen und Beschäftigten der Nordwestschweiz im Jahr 1998 berechnet. Diese neuen Anteile der Gemeinden werden aber weiterhin auf den Gesamtbestand der beiden Grössen im Jahr 1991 bezogen. So ergeben sich die fiktiven Erwerbspersonen- und Beschäftigtenzahlen $\left(\mathrm{EP}_{\mathrm{i}}^{*}, \mathrm{~B}_{\mathrm{i}}{ }^{*}\right)$ der Gemeinden im Jahr 1998 für in der Summe konstante Erwerbspersonenund Beschäftigtenzahlen in der Nordwestschweiz (s. Beispiel).

Berechnungsbeispiel der konjunkturbereinigten Standortdivergenz für die Gemeinde i:

$\mathrm{D}_{\mathrm{i}}^{*}=\left|\mathrm{EP}_{\mathrm{i}}^{*}-\mathrm{B}_{\mathrm{i}}^{*}\right|$

mit

$E P_{i}^{*}=\frac{E P_{i 1998}}{E P_{n w c h 1998}} \times E P_{n w c h 1991}$

$B_{i}^{*}=\frac{B_{i 1998}}{B_{n w c h 1998}} \times B_{n w c h 1991}$

mit $E P_{n w c h}, B_{n w c h}=$ Bestandsgrössen für die Nordwestschweiz insgesamt.

Die so berechneten Werte geben an, wie sich die lokale Erwerbspersonen- oder Beschäftigtenzahl verändert hätte, wenn es lediglich die beobachteten räumlichen Verschiebungen der Anteile an Erwerbspersonen und Beschäftigten gegeben hätte, nicht aber die konjunkturell bedingten Unterschiede der Arbeitsplatz- und
Erwerbspersonenzahlen in der Nordwestschweiz insgesamt.

\section{Ergebnisse}

Basel verliert nicht nur bei den Einwohnerzahlen, sondern auch bei den Arbeitsplatzzahlen an Bedeutung gegenüber dem Umland. Im Folgenden werden kurz die wichtigsten Kennzahlen der Verlagerung der Wachstumsdynamik beider Grössen ins Umland dargestellt (Kap. 4.1), bevor in Kapitel 4.2 und 4.3 die Auswirkungen dieser Vorgänge auf die Mischung von Erwerbspersonen und Beschäftigten auf Gemeindeund Bezirksebene quantifiziert werden.

\section{1 Übersicht - Ausmass und Hauptgebiete des Umlandwachstums von Wohnen und Arbeiten}

Bedeutungsgewinn des Umlandes bei den Bevölkerungszahlen

Der Anteil der Stadt Basel am Einwohnerbestand der Nordwestschweiz ging von 1991 bis 1998 um $1.7 \%$-Punkte auf $32.0 \%$ zurück. Insgesamt nahm die Bevölkerungszahl im Untersuchungszeitraum um 2.2\% zu. Eine räumliche Aufteilung der Nordwestschweiz in Kern, Agglomerationsgürtel und übrige Nordwestschweiz (vgl. Karte 1) gibt einen ersten Überblick über die räumliche Verteilung dieser Umschichtungen (Tab.1): Ausgehend vom Zentrum Basel, welches im Untersuchungszeitraum über 5000 oder $3 \%$ seiner Einwohnerschaft verloren hat, nahm die Zuwachsrate der Einwohnerzahl mit der Entfernung von Basel zu: Während der innere Agglomerationsbereich kaum mehr Bevölkerungszuwachs verzeichnet $(+0.3 \%)$, ist das Wachstum im äusseren Agglomerationsgürtel $(+5.4 \%)$ und vor allem in der übrigen Nordwestschweiz $(+10.5 \%)$ beträchtlich.

Ein höheres Wachstum der Bevölkerungszahlen im Umland sollte gemäss dem herkömmlichen Leitbild der dezentralen Konzentration vor allem die Subzentren mit tragfähiger Infrastruktur und guter Anbin-

\begin{tabular}{|l|r|r|r|r|r|r|}
\hline \multicolumn{1}{|c|}{ Teilgebiete der } & \multicolumn{2}{|c|}{ Einwohner } & \multicolumn{2}{c|}{ Änderung 1991-1998 } & \multicolumn{2}{c|}{ Anteil } \\
Nordwestchweiz & 1991 & 1998 & absolut & $(\%)$ & 1991 & 1998 \\
\hline Zentrum & 178498 & 173216 & -5282 & $-3,0$ & 33,7 & 32,0 \\
Innere Agglomeration & 124545 & 124914 & 369 & 0,3 & 23,5 & 23,1 \\
Äussere Agglomeration & 137856 & 145281 & 7425 & 5,4 & 26,1 & 26,9 \\
Übrige Nordwestschweiz & 88263 & 97527 & 9264 & 10,5 & 16,7 & 18,0 \\
\hline Nordwestschweiz & 529162 & 540938 & 11776 & 2,2 & 100,0 & 100,0 \\
\hline
\end{tabular}

Tab. 1: Veränderung der Bevölkerungszahlen in Teilgebieten der Nordwestschweiz

Changes in population figures in regional units of North-West Switzerland

Evolution du nombre d'habitants dans des régions du nord-ouest de la Suisse

Quelle: Statistik des jährlichen Bevölkerungsstandes (ESPOP) 1991, 1998 


\begin{tabular}{|c|c|c|c|r|r|r|}
\hline $\begin{array}{l}\text { Gemeindegrösse } \\
\text { nach Bevölke- } \\
\text { rungszahl }\end{array}$ & $\begin{array}{c}\text { Bevölkerung } \\
1991\end{array}$ & $\begin{array}{c}\text { Bevölkerung } \\
1998\end{array}$ & \multicolumn{2}{|l|}{ Änderung 1991-1998 } & \multicolumn{2}{|c|}{ Anteil an NWCH (\%) } \\
& 178498 & 173216 & -5282 & $-3,0$ & 33,7 & 32,0 \\
\hline über 100000 & 137431 & 136835 & -596 & $-0,4$ & 26,0 & 25,3 \\
10000 bis 99999 & 166376 & 178564 & 12188 & 7,3 & 31,4 & 33,0 \\
1000 bis 9999 & 46857 & 52323 & 5466 & 11,7 & 8,9 & 9,7 \\
\hline bis 999 & 529162 & 540938 & 11776 & 2,2 & 100,0 & 100,0 \\
\hline
\end{tabular}

Tab. 2: Veränderung der Bevölkerungszahlen nach Gemeindegrössenklassen Changes in population figures, by community and size class Evolution du nombre d'habitants par classe de grandeur des communes Quelle: Statistik des jährlichen Bevölkerungsstandes (ESPOP) 1991, 1998

dung ans Verkehrsnetz stärken. Tatsächlich verlieren aber nicht nur der grosse Einwohner- und Arbeitsplatzschwerpunkt Basel sondern - zumindest per Saldo - auch mittelgrosse Gemeinden relativ an Bedeutung (Tab. 2). Höchste Zuwachsraten können die kleinsten Gemeinden verbuchen. Bei einem Einwohnerzuwachs der Nordwestschweiz von $2.2 \%$ verzeichneten die kleinsten Wohngemeinden mit unter 1000 Einwohnern ein Wachstum von $11.7 \%$.

Bedeutungsgewinn des Umlandes bei den Beschäftigtenzahlen

Der Anteil von Basel an den Beschäftigten in der Nordwestschweiz verringerte sich um $2.9 \%$-Punkte auf 49.1\%. Auch bei den Arbeitsplätzen zeigt die Unterscheidung von Agglomerationskern, innerer, äusserer Agglomeration und übriger Nordwestschweiz die ungünstigste Veränderung für den Agglomerationskern (-14.2\%) (Tab. 3). Bei einer insgesamt zurückgehenden Arbeitsplatzzahl in der Nordwestschweiz (-9.2\%) äussern sich räumliche Gewichtsverschiebungen vor allem durch unterschiedlich starken Arbeitsplatzverlust. Im
Gegensatz zur Bevölkerungsdynamik weist bei den Arbeitsplätzen der innere Agglomerationsbereich die relativ günstigste Veränderung auf (lediglich $-3.2 \%$ ). Der äussere Agglomerationsbereich $(-3.7 \%)$ und vor allem die übrige Nordwestschweiz $(-5.1 \%)$ verzeichnen ebenfalls unterdurchschnittliche Rückgänge.

Auch bei den Beschäftigtenzahlen kommt die günstigere Entwicklung im Umland nicht den regionalen Subzentren zugute (Tab. 4): Lediglich die kleinsten Arbeitsplatzgemeinden mit weniger als 500 Beschäftigten erreichten trotz eines Arbeitsplatzrückgangs in der Nordwestschweiz als Ganzes von $-9 \%$ noch einen Zuwachs von $2 \%$.

Unterschiedliche räumliche Wachstumsschwerpunkte von Bevölkerung und Beschäftigung

Schon obiger grober Überblick zeigt, dass die allgemeine Tendenz des grösseren Wachstums im Umland nicht zur Stärkung der regionalen Subzentren führt, wie es das Leitbild der dezentralen Konzentration anstrebt. Darüber hinaus wird deutlich, dass die aktuelle Dynamik von Bevölkerung und Beschäftigung in der Fläche

\begin{tabular}{|l|r|r|r|r|r|r|}
\hline \multicolumn{1}{|c|}{ Teilgebiete der } & \multicolumn{2}{|c|}{ Beschäftigte } & \multicolumn{2}{c|}{ Änderung 1991-1998 } & \multicolumn{2}{c|}{ Anteil } \\
Nordwestschweiz & 1991 & 1998 & absolut & $(\%)$ & 1991 & 1998 \\
\hline Zentrum & 168397 & 144470 & -23927 & $-14,2$ & 52,0 & 49,1 \\
Innere Agglomeration & 55942 & 54150 & -1792 & $-3,2$ & 17,3 & 18,4 \\
Äussere Agglomeration & 65209 & 62781 & -2428 & $-3,7$ & 20,1 & 21,3 \\
Übrige Nordwestschweiz & 34590 & 32843 & -1747 & $-5,1$ & 10,7 & 11,2 \\
\hline Nordwestschweiz & 324138 & 294244 & -29894 & $-9,2$ & 100,0 & 100,0 \\
\hline
\end{tabular}

Tab. 3: Veränderung der Beschäftigtenzahlen in Teilgebieten der Nordwestschweiz

Changes in employment in regional units of North-West Switzerland

Evolution du nombre d'emplois dans des régions du nord-ouest de la Suisse

Quelle: Betriebszählung 1991, 1998 


\begin{tabular}{|c|c|c|c|c|c|c|}
\hline \multirow{2}{*}{$\begin{array}{l}\text { Gemeindegrösse } \\
\text { nach Beschäftig- } \\
\text { tenzahl }\end{array}$} & \multirow{2}{*}{$\begin{array}{c}\text { Beschäftigte } \\
1991\end{array}$} & \multirow{2}{*}{$\begin{array}{c}\text { Beschäftigte } \\
1998\end{array}$} & \multicolumn{2}{|c|}{ Änderung 1991-1998 } & \multicolumn{2}{|c|}{ Anteil an $\mathrm{NWCH}(\%)$} \\
\hline & & & Beschäftigte & $(\%)$ & 1991 & 1998 \\
\hline über 50000 & 168397 & 144470 & -23927 & $-14,2$ & 52,0 & 49,1 \\
\hline 5000 bis 49999 & 66313 & 63558 & -2755 & $-4,2$ & 20,5 & 21,6 \\
\hline 500 bis 4999 & 73051 & 69553 & -3498 & $-4,8$ & 22,5 & 23,6 \\
\hline 0 bis & 16377 & 16663 & 286 & 1,7 & 5,1 & 5,7 \\
\hline & 324138 & 294244 & -29894 & $-9,2$ & 100,0 & 100,0 \\
\hline
\end{tabular}

Tab. 4: Veränderung der Beschäftigtenzahlen nach Gemeindegrössenklassen

Changes in employment, by community and size class

Evolution du nombre d'emplois par classe de grandeur des communes

Quelle: Betriebszählung 1991, 1998

nicht immer die gleichen Orte betrifft: Während Gemeinden mit starker positiver Abweichung der Bevölkerungszunahme vor allem im äusseren Agglomerationsbereich und der übrigen Nordwestschweiz vorkommen, weisen bei den Beschäftigtenzahlen eher zentrumsnahe Gemeinden im inneren Agglomerationsgürtel positive Abweichungen vom Entwicklungstrend auf. Selbst innerhalb der verschiedenen Agglomerationsgürtel sind die Veränderungen nicht homogen. Die Gemeindeebene (Karte 2) zeigt, dass das oberste Quartil (Gemeinden mit besonders grossem Bevölkerungszuwachs) nur selten auch einen grossen Zuwachs der Beschäftigtenzahlen aufweist. Die Korrelation der gemeindlichen Wachstumsraten von Einwohnern und Arbeitsplätzen für die Nordwestschweiz ergibt auch keinen signifikanten Zusammenhang $\left(\mathrm{R}^{2}\right.$ $=0.007$; Irrtumswahrscheinlichkeit Sig. $>10 \%$ ). Offenbar gelten unterschiedliche Standortanforderungen von Wohnen und Arbeiten auch für suburbane und ländliche Standorte.

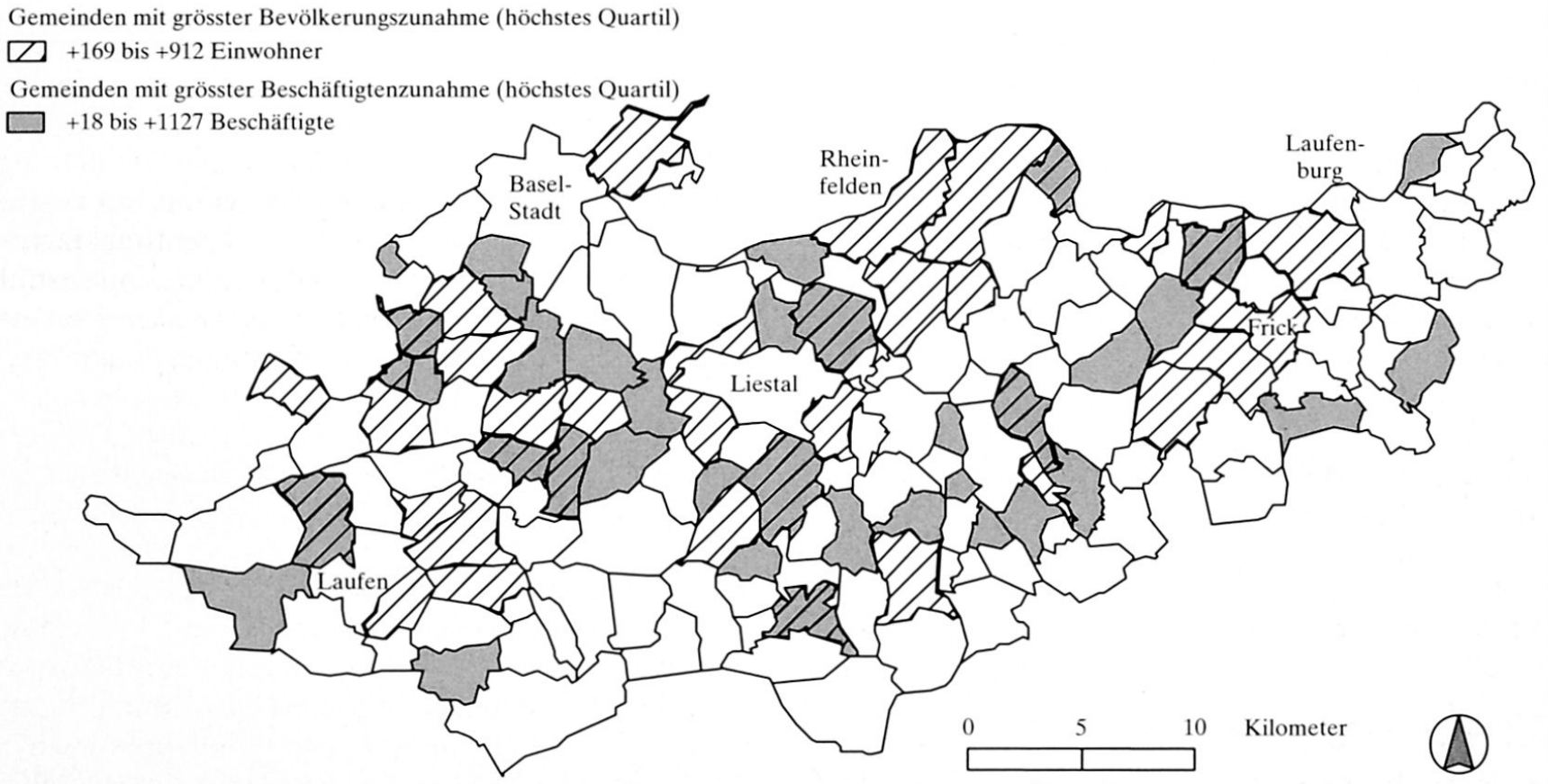

Karte 2: Gemeinden mit dem grössten Zuwachs der Bevölkerung und Beschäftigung Communes with the highest population and employment growth rates

Communes présentant les plus grandes augmentations du nombre d'habitants et d'emplois

Quelle: Statistik des jährlichen Bevölkerungsstandes (ESPOP) 1991, 1998; Betriebszählung 1991, 1998; Kartengrundlage: BFS GEOSTAT/L+T; Kartographie und Bearbeitung: R. VoLMAN 


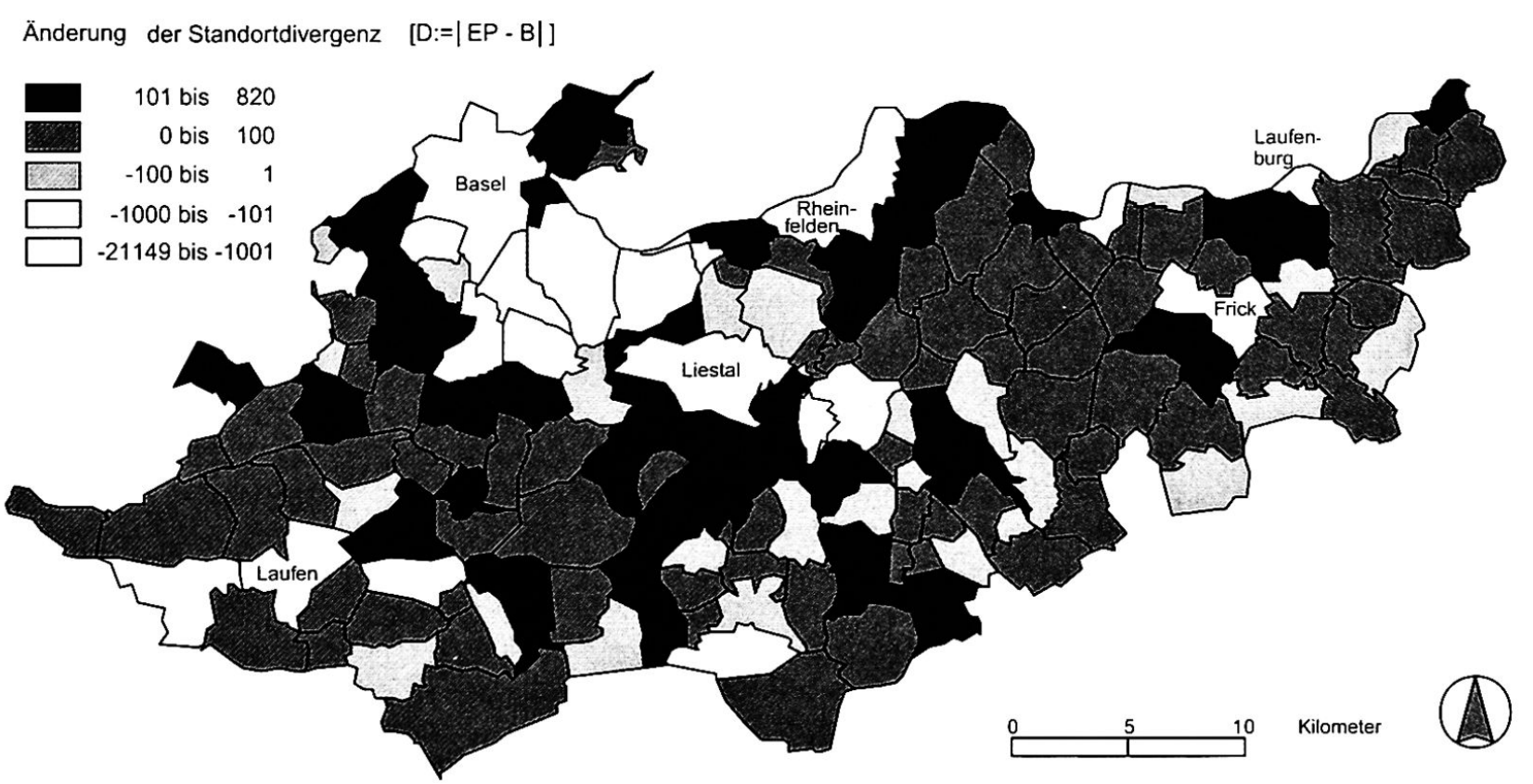

Karte 3: Änderung der Standortdivergenzen 1991-1998

Changes in "site divergence»1991-1998

Evolution des divergences d'emplacement de 1991 à 1998

Quelle: Statistik des jährlichen Bevölkerungsstandes (ESPOP) 1991, 1998; Betriebszählung 1991, 1998; Kartengrundlage: BFS GEOSTAT/L+T; Kartographie und Bearbeitung: R. VoLmaN

Beispiel: Die Standortdivergenz - also die Anzahl der vor Ort «fehlenden» oder «überschüssigen» Erwerbspersonen - hat sich in Laufen um 101 bis 1000 verringert.

Example: "Site divergence», i.e. the number of employed persons either lacking or in surplus locally, decreased in Laufen by 101 to 1000.

Exemple: A Laufon, la divergence d'emplacement - c'est-à-dire le nombre de personnes actives «manquantes»ou "excédentaires» - s'est abaissée entre 101 et 1000.

4.2 Wirkung des Umlandwachstums auf die Mischung von Wohnen und Arbeiten auf Gemeindeebene

Kurzfristig ausgewogenere Mischung von Erwerbspersonen und Beschäftigten auf Gemeindeebene

Die Wirkung der räumlich unterschiedlichen Wachstumsschwerpunkte von Bevölkerung und Beschäftigung auf die Ausgewogenheit der Mischung von Wohnen und Arbeiten kann mit der in Kap. 3.2 beschriebenen Grösse «Standortdivergenz» operationalisiert werden. Je weniger «fehlende» Erwerbspersonen oder Beschäftigungsmöglichkeiten es in einem Gebiet gibt, desto geringer wird dieser Indikator.

Die räumliche Umverteilung von Erwerbspersonen und Beschäftigten führte von 1991 bis 1998 über alle Gemeinden der Nordwestschweiz aufsummiert immerhin zu einer Verringerung der Standortdivergenz um $-11.13 \%$. Die Anzahl der vor Ort «fehlenden» Erwerbspersonen oder Beschäftigungsmöglichkeiten ging von $151^{\prime} 710$ auf $134^{\prime} 829$ zurück. Die Auswirkungen sind aber von Gemeinde zu Gemeinde unterschiedlich. Eine ausgewogenere Mischung stellte sich vor allem im Kern und inneren Bereich der Agglomeration zwischen Basel und Liestal ein sowie in Subzentren mit bisher überschüssigem Angebot an Arbeitsplätzen wie beispielsweise Laufen, Rheinfelden, Laufenburg oder Frick (Karte 3). Die bevölkerungsmässig stark wachsenden Gemeinden im äusseren Agglomerationsbereich und in der übrigen Nordwestschweiz müssen dagegen meist eine Zunahme ihrer lokalen Differenzen zwischen Erwerbspersonen- und Beschäftigtenzahl verbuchen.

Konjunkturbereinigt kaum Änderungen der Mischung von Erwerbspersonen und Beschäftigten

Die obigen Ergebnisse sind von der schlechten wirtschaftlichen Konjunktur während des Untersuchungszeitraums von 1991 bis 1998 geprägt: Während sich die Anzahl der Beschäftigten regionsweit aussergewöhnlich verringerte, nahm die Einwohnerzahl weiter zu, so dass es für Gemeinden mit schon bestehendem Defizit an Arbeitsplätzen in dieser Phase besonders schwierig war, die örtliche Standortdivergenz abzubauen. Gemeinden mit bestehendem Arbeitsplatzüberschuss verringerten ihren Mangel an Erwerbspersonen auch 


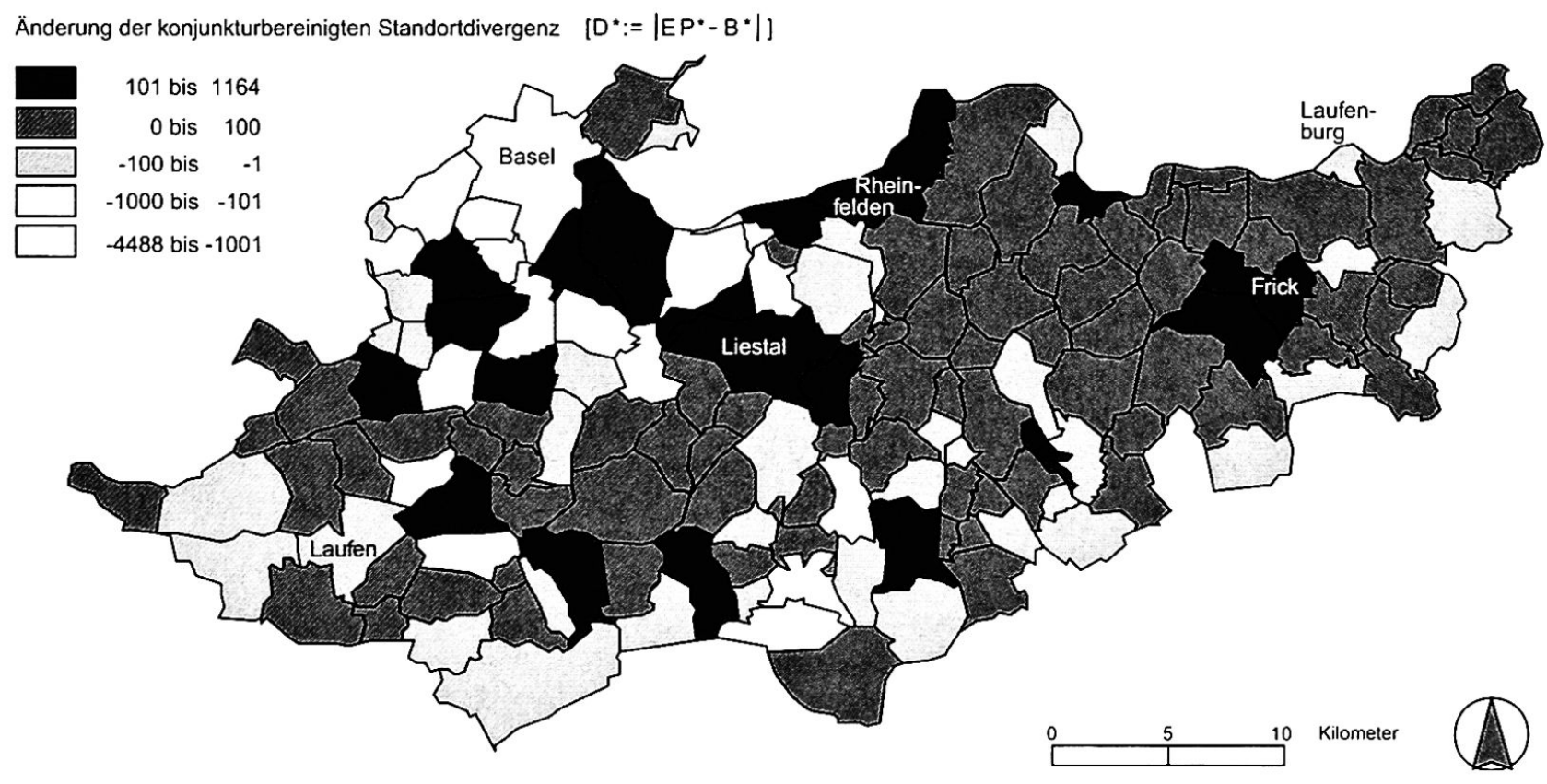

Karte 4: Konjunkturbereinigte Änderung der Standortdivergenzen 1991-1998

Changes in «site divergence» adjusted for economic growth trends 1991-1998

Evolution des divergences d'emplacement (avec ajustement des variations conjoncturelles) 1991-1998

Quelle: Statistik des jährlichen Bevölkerungsstandes (ESPOP) 1991, 1998; Betriebszählung 1991, 1998; Kartengrundlage: BFS GEOSTAT/L+T; Kartographie und Bearbeitung: R. VoLMAN

Beispiel: Unter Ausblendung der konjunkturellen Einflüsse hätte sich die Standortdivergenz - also die Anzahl der vor Ort «fehlenden» oder «überschüssigen» Erwerbspersonen - in Laufen um 1 bis 100 verringert.

Example: "Site divergence» in Laufen, i.e. the number of employed persons either lacking or in surplus locally, would have decreased by 1 to 100 if the influence of economic cycles is mathematically adjusted.

Exemple: A Laufon, la divergence d'emplacement - c'est-à-dire les personnes actives «manquantes» ou «supplémentaires» - se serait abaissée entre 1 et 100 avec un ajustement des influences conjoncturelles.

schon im Zuge des konjunkturell bedingten, generellen Arbeitsplatzabbaus. Zur Abschätzung der langfristigen Wirkungen der Verlagerung der Wachstumsdynamik von Erwerbspersonen und Beschäftigten ins Umland auf die Nutzungsmischung ist es notwendig, die Einflüsse der gerade vorherrschenden Wachstumstrends von Einwohner- und Arbeitsplatzzahlen auf die räumliche Mischung von Erwerbspersonen und Arbeitsplätzen auszublenden. Dies wird durch die Berechnung der in Kap. 3.2 beschriebenen konjunkturbereinigten Standortdivergenz erreicht, welche nur die räumliche Verlagerung der Anteile an Erwerbspersonen und Beschäftigten beachtet, nicht aber die gerade vorherrschenden Wachstumsraten von Bevölkerung und Beschäftigung insgesamt.

Die aufsummierten Standortdivergenzen der Gemeinden gingen bei konjunkturbereinigter Betrachtung von 1991 bis 1998 nur um 1.02\% zurück. In der langfristigen Perspektive über konjunkturelle Schwankungen hinweg würden die aktuellen Trends der räumlichen Verlagerung der Wachstumsschwerpunkte von Bevölkerung und Beschäftigung ins Umland also nur eine sehr bescheidene Verbesserung der Funktionsmischung auf Gemeindeebene bewirken. Das Leitbild der dezentralen Nutzungsmischung in der Fläche auf Gemeindeebene wird somit durch das gegenwärtige Bevölkerungs- und Beschäftigungswachstum im Umland nicht unterstützt. Immerhin wäre aber per Saldo auch keine Zunahme der lokalen Differenzen zwischen Erwerbspersonenund Beschäftigtenzahl zu erwarten. Die Darstellung der Veränderungen der gemeindlichen Standortdivergenzen in Karte 4 zeigt, dass es konjunkturbereinigt nur in folgenden Gemeinden eine deutliche Verringerung der lokalen Differenzen zwischen Erwerbspersonen und Beschäftigten gab:

In den älteren Arbeitsplatzstandorten Basel und Pratteln im Kernbereich der Agglomeration ist die ausgewogenere Mischung Folge eines allgemeinen Bedeutungsverlustes, der im Bereich der Beschäftigtenzahlen gravierender ausfällt als bei den Erwerbspersonenzahlen (Tab. 5). In den kleinen, im weiteren Umland gelegenen Industriezentren Zwingen (Papierindustrie), Waldenburg (Feinmechanik) und Breitenbach führt die zunehmende Bedeutung als Wohnstandort bei 


\begin{tabular}{|c|c|c|c|}
\hline Gebietstyp & $\begin{array}{l}\text { Gemeinden mit starker } \\
\text { Verbesserung der lokalen } \\
\text { Funktionsmischung } \\
\text { (Abbau der Standortdivergenzen } \\
\text { um mehr als } 100 \text { Erwerbspersonen) }\end{array}$ & Prozesse & Auswirkung \\
\hline $\begin{array}{l}\text { Traditionelle } \\
\text { Industriestandorte in } \\
\text { der inneren } \\
\text { Agglomeration }\end{array}$ & Basel, Pratteln & $\begin{array}{l}\text { Arbeitsplatzabbau grösser als } \\
\text { Abnahme der } \\
\text { Erwerbspersonen }\end{array}$ & \multirow[t]{2}{*}{$\begin{array}{l}\text { Abbau der Defizite von } \\
\text { Erwerbspersonen im } \\
\text { Verhältnis zu den } \\
\text { Arbeitsplätzen }\end{array}$} \\
\hline $\begin{array}{l}\text { Ländliche Industrie- } \\
\text { und } \\
\text { Gewerbegemeinden }\end{array}$ & Zwingen, Breitenbach, Waldenburg & $\begin{array}{l}\text { Arbeitsplatzabbau und } \\
\text { Zunahme der } \\
\text { Erwerbspersonen }\end{array}$ & \\
\hline $\begin{array}{l}\text { Suburbane } \\
\text { Gemeinden in der } \\
\text { inneren } \\
\text { Agglomeration }\end{array}$ & $\begin{array}{l}\text { Allschwil, Binningen, Bottmingen, } \\
\text { Ettingen, Reinach, Arlesheim, } \\
\text { Gempen, Füllinsdorf }\end{array}$ & $\begin{array}{l}\text { Arbeitsplatzzunahme und } \\
\text { Stagnation der } \\
\text { Erwerbspersonenzahl }\end{array}$ & \multirow[t]{2}{*}{$\begin{array}{l}\text { Abbau von } \\
\text { Arbeitsplatzdefiziten im } \\
\text { Verhältnis zu den Erwerbs- } \\
\text { personen }\end{array}$} \\
\hline Ländliche Gemeinden & Oberdorf (BL) & $\begin{array}{l}\text { Arbeitsplatzzunahme grösser } \\
\text { als Zunahme der } \\
\text { Erwerbspersonen }\end{array}$ & \\
\hline
\end{tabular}

Tab. 5: Beispiele für starke Rückgänge lokaler Standortdivergenzen

Examples of a strong decline in local site divergence, and the processes involved

Exemples de forts reculs des divergences d'emplacement locales et processus sous-jacents

gleichzeitigem Rückgang der Industrie dazu, dass sich die Zahlen von Beschäftigten und Erwerbspersonen einander annähern. Eine ausgewogenere Mischung von Wohnen und Arbeiten ohne Schrumpfungsprozesse verzeichnet der Ring kleiner Gemeinden um den Agglomerationskern und das ländliche Oberdorf.

Deutliche Zunahmen der Differenzen zwischen Erwerbspersonen- und Beschäftigtenzahlen zeigen sich bei konjunkturbereinigter Betrachtung vor allem in den bestehenden Arbeitsplatzzentren in der Nähe von Basel (Münchenstein, Muttenz, Liestal, Kaiseraugst und Rheinfelden oder in Frick an der neuen Autobahn nach Zürich (Karte 4). Die zum Teil geringe Anziehungskraft dieser Gemeinden als Wohnstandort und die attraktive Lage für Arbeitsplätze - Nähe zu Basel und gute Verkehrsanschlüsse - verstärkt die Spezialisierung als Arbeitsplatzgemeinden. Auch in den meisten Gemeinden im äusseren Agglomerationsgürtel und im Umland der Agglomeration nehmen die Differenzen zwischen Erwerbspersonen- und Beschäftigtenzahlen zu. Dort wächst einseitig die Zahl der Erwerbspersonen. Gerade in eher abgelegenen Gemeinden, wo eine fehlende lokale Mischung von Erwerbspersonen und Beschäftigungsmöglichkeiten möglicherweise mit besonders langen Arbeitswegen bezahlt werden muss, unterbleibt eine ausgewogenere Mischung von Arbeiten und Wohnen.

\subsection{Wirkung des Umlandwachstums auf die Mischung von Wohnen und Arbeiten auf Bezirksebene}

Kapitel 4.2 hat gezeigt, dass eine dezentrale Nutzungsmischung auf Gemeindeebene durch das Umland- wachstum kaum zu erwarten ist. Es wäre aber zu untersuchen, ob das Umlandwachstum zumindest auf mesoskaliger Ebene der Bezirke mit einer Nutzungsmischung in der Fläche einhergeht. Im Sinne einer Verringerung des Verkehrsaufkommens wäre es als zweitbeste Lösung noch akzeptabel, wenn sich eine Mischung von Wohnen und Arbeiten zumindest innerhalb benachbarter Gruppen von Gemeinden wie den Bezirken einstellt. Immerhin könnten die Arbeitswege dann auf diese Räume kleiner Ausdehnung beschränkt bleiben und müssten sich nicht über die ganze Nordwestschweiz erstrecken wie dies z.B. beim Pendeln vom Umland nach Basel der Fall wäre.

Die Veränderung der Standortdivergenz auf Bezirksebene wurde gemäss der Beschreibung in Kap. 3.2 berechnet. Es werden also nur noch solche Differenzen zwischen Erwerbspersonen- und Beschäftigtenzahl erfasst, die nicht innerhalb eines Bezirkes ausgeglichen werden können. Auf der grösseren Untersuchungsebene der Bezirke führen die neuen Standortmuster von Wohnen und Arbeiten auch in der konjunkturbereinigten Betrachtung zu einer ausgewogeneren räumlichen Mischung von Erwerbspersonen und Beschäftigten. Die räumliche Umverteilung von Erwerbspersonen- und Beschäftigtenzahlen führte von 1991 bis 1998 zu einer Verringerung der Summe der Standortdivergenzen der Bezirke in der Nordwestschweiz um $8.87 \%$ (ohne Konjunkturbereinigung verringert sich die Summe der Standortdivergenzen sogar um $13.88 \%$ ). In nur sieben Jahren hat sich die Anzahl der rein rechnerisch bestehenden Defizite an Erwerbstätigen oder Beschäftigten innerhalb der Bezirke um fast ein Zehntel verringert. 


\begin{tabular}{|l|r|r|r|r|}
\hline \multirow{2}{*}{ Bezirk*) } & \multicolumn{3}{|c|}{ Konjunkturbereinigter } & Änderung der Standortdivergenz \\
& \multicolumn{2}{|c|}{$\begin{array}{c}\text { Arbeitsplatzüberschuss } \\
\text { 1991-1998 }\end{array}$} \\
\hline Dorneck & 1991 & 1998 & \multicolumn{2}{|c|}{ absolut } \\
Thierstein & -2113 & -2177 & 64 & 3,0 \\
Basel-Stadt & -1501 & -1807 & 304 & 20,4 \\
Arlesheim & 68383 & 63845 & -4538 & $-6,6$ \\
Laufen & -10662 & -5739 & -4923 & $-46,2$ \\
Liestal & -959 & -1556 & 597 & 62,2 \\
Sissach & 2856 & 3108 & 252 & 8,8 \\
Waldenburg & -4767 & -4965 & 198 & 4,2 \\
Laufenburg & -2401 & -2478 & 77 & 3,2 \\
Rheinfelden & -1746 & -2140 & 395 & 22,6 \\
\hline Nordwestschweiz & -1291 & -291 & -1000 & $-77,5$ \\
\hline
\end{tabular}

*) Anmerkung: Die Bezirksexklaven Kleinlützel, Rodersdorf, Metzerlen, Hofstetten, Witterswil oder Bättwil sind den jeweils am besten erreichbaren, angrenzenden Bezirken zugeschrieben, Bözen, Elfingen und Effingen dem Bezirk Laufenburg.

Tab. 6: Veränderung der Standortdivergenzen auf Bezirksebene

Changes of site divergence at district level

Evolution des divergences d'emplacement par district

Quelle: Statistik des jährlichen Bevölkerungsstandes (ESPOP) 1991; Betriebszählung 1991, 1998

Wenn auch nicht kleinsträumig auf Gemeindeebene, so führt die Verlagerung des Bevölkerungs- und Beschäftigungswachstums vom Zentrum Basel ins Umland doch zumindest auf Bezirksebene per Saldo zu einer erheblichen Verbesserung der Mischung von Arbeiten und Wohnen.

Eine räumliche Aufgliederung dieser Wirkung zeigt, dass nicht alle Bezirke in gleicher Weise von einer ausgewogeneren Nutzungsmischung profitieren (Tabelle 6). Vor allem die dem Agglomerationskern Basel nahen Bezirke Arlesheim und Rheinfelden können ihre Differenzen zwischen Erwerbspersonen- und Beschäftigtenzahlen durch einen Abbau ihrer Arbeitsplatzdefizite beträchtlich reduzieren. Laufen, Thierstein, Waldenburg, Sissach und Laufenburg müssen dagegen eine Vergrösserung ihres Arbeitsplatzdefizits hinnehmen. Trotz der besseren Mischung von Arbeiten und Wohnen auf Bezirksebene für die Nordwestschweiz insgesamt erfahren die randständigen Bezirke der Nordwestschweiz mit ihren hohen Wachstumsraten der Einwohnerzahlen also selbst auf der weiträumigeren Bezirksebene keine Verbesserung der Nutzungsmischung.

\section{Fazit}

Die räumliche Umschichtung von Bevölkerung und Beschäftigung führte von 1991 bis 1998 zu einem relativen Bedeutungsverlust des Agglomerationszentrums Basel zugunsten des Umlandes. Dabei unterscheiden sich die räumlichen Muster des Wachstums von Bevölkerung und Beschäftigung im Umland. Per Saldo entsteht besonders unter Ausschaltung konjunkturbedingter Verzerrungen auf Gemeindeebene kaum eine dezentrale Funktionsmischung. Erst auf der Ebene von Bezirken ist eine spürbare Verbesserung der Funktionsmischung von 1991 bis 1998 erkennbar. $\mathrm{Zu}$ einer ausgewogeneren Mischung von Erwerbspersonen und Beschäftigten kommt es vor allem in den Bezirken im Bereich der Agglomeration. In den von Basel weiter entfernten Bezirken nehmen die Differenzen zwischen Erwerbspersonen und Beschäftigten durch die einseitige Zunahme der Bevölkerungszahlen ohne Zugewinn an Beschäftigung zu.

Für die Vorstellung einer dezentralen Nutzungsmischung in der Fläche heisst dies, dass zumindest die dezentrale Mischung auf kleinsträumiger Ebene wie Gemeinden oder sogar Quartieren nicht von den gegenwärtigen Mechanismen der Standortwahl von Wohnen und Arbeiten unterstützt wird. Trotz rückläufiger Bedeutung der industriellen Fertigung sind die Standortansprüche von Wohnen und Arbeiten offenbar noch so verschieden, dass unterschiedliche Gemeinden bevorzugt werden. Zumindest innerhalb der Agglomeration sind aber Ansätze einer dezentralen Mischung von Wohnen und Arbeiten in der Fläche auf Bezirksebene erkennbar. Daraus könnte unter dem Gesichtspunkt der Minimierung des Verkehrsaufkommens eine zweigeteilte Strategie abgeleitet werden: Für periurbane Gebiete wäre eine striktere Umsetzung des Leitbildes der dezentralen Konzentration in wenigen Zentren mit guter Einbindung in den öffentlichen Verkehr zu verfolgen. Im Perimeter der 
Agglomeration könnte auf eine strenge Lenkung von Wohnen und Arbeiten auf Zentren und Subzentren verzichtet werden. Stattdessen sollten die vorhandenen Ansätze zur dezentralen Nutzungsmischung in der Fläche durch nutzungsvariable Bauzonen und den Ausbau kleinräumiger Netze des öffentlichen Verkehrs, die nicht nur auf die radialen Zubringerdienste zum Zentrum Basel ausgerichtet sind, begleitet werden.

\section{Literatur}

Apel, D., Henckel, D. et al. (1995): Flächen sparen, Verkehr reduzieren: Möglichkeiten zur Steuerung der Siedlungs- und Verkehrsentwicklung. $-=$ Difu-Beiträge zur Stadtforschung 16, Berlin.

Bundesamt Für RaUmPLANUNG \& EIDgenÖSSISCHES Justiz- Und Polizeidepartement (1996): Bericht über die Grundzüge der Raumordnung Schweiz.- Bern.

Christaller, W. (1933): Die zentralen Orte in Süddeutschland. Eine ökonomisch-geographische Untersuchung über die Gesetzmässigkeit der Verbreitung und Entwicklung der Siedlungen mit städtischen Funktionen. - Jena: G. Fischer.

Göderitz, J., Roland, R. \& H. Hoffmann (1957): Die gegliederte und aufgelockerte Stadt. $-=$ Archiv für Städtebau und Landesplanung 4, Tübingen.

Hesse, M. \& S. Schmitz (1998): Stadtentwicklung im Zeichen von "Auflösung» und «Nachhaltigkeit». - In: Informationen zur Raumentwicklung 7/8: 435-453.

HILPERT, T. (1988): Le Corbusiers "Charta von Athen»: Texte und Dokumente. - = Bauwelt Fundamente 56, Braunschweig, Wiesbaden.

Howard, E. (1907): Gartenstädte in Sicht. - Jena: Dietrichs; übersetzt von M. Wallroth-Unterlip.

IsBary, G., VON DER HEIDE, H.-J. \& G. MülleR (1969): Gebiete mit gesunden Strukturen und Lebensbedingungen. Merkmale und Abgrenzung. - = AKADEmIE FÜR RAUMFORSCHUNG UND LANDESPLANUng (Hrsg.): Abhandlungen 57, Hannover.

KAHNERT, R. (1998): Wirtschaftsentwicklung, Sub- und Desurbanisierung. - In: BUNDESAMT FÜR BAUWESEN UND RAUmordnung (Hrsg.): Stadt - Landschaft. $=$ Informationen zur Raumentwicklung 7/8, Bonn: 509-520.

KNOFLACHER, H. (1995): Ersticken unsere Siedlungen im Verkehr? - = Mobilität und Verkehr, 4. Luzerner Umwelt-Symposium 1994, Luzern.

KutTER, E. \& A. STEIN (1996): Verkehrsminderung «vor Region» - von der Idee zur Umsetzung. - In: BundesAMT Für BAUWESEN UND RAUMORdNung (Hrsg.): Ballungsraumverkehr - Integrierte Verkehrsplanung und Verkehrsvermeidung. - = Informationen zur Raumentwicklung 7/8, Bonn: 461-488.

OPASCHOWSKI,H.W.(1999): Umwelt, Freizeit, Mobilität: Konflikte und Konzepte. - 2. Aufl., Opladen: Leske + Budrich.
Schuler, M., Huissod, T., Jemelin, C. \& S. Stofer (1997): Strukturatlas der Schweiz. - Zürich: NZZ.

SieverTs, T. (1997): Zwischenstadt - zwischen Ort und Welt, Raum und Zeit, Stadt und Land. - = Bauwelt Fundamente 118, Braunschweig, Wiesbaden.

WÜRDEMANN, G. (1998): Handlungsfelder der räumlichen Planung für eine lebenswerte und verkehrssparsame Stadt und Region. - In: BundESAMT FÜR BAUWESEN UND RAUMORDNUNG (Hrsg.): Strategien für einen raum- und umweltverträglichen Verkehr - = Informationen zur Raumentwicklung 6, Bonn: 351-368.

\section{Zusammenfassung: Suburbanes Wachstum von Bevöl-} kerung und Beschäftigung als Weg zur dezentralen Mischung von Wohnen und Arbeiten? - Eine empirische Untersuchung am Beispiel der Nordwestschweiz

In der Nordwestschweiz verlagern sich die räumlichen Schwerpunkte des Bevölkerungs- und Arbeitsplatzwachstums weg vom Agglomerationskern Basel ins Umland. Davon profitieren weniger die regionalen Subzentren, als die kleinen Gemeinden. Diese Suburbanisierungsvorgänge haben auch Auswirkungen auf die räumliche Zuordnung von Wohn- und Arbeitsstätten. Aus Sicht der Verkehrsvermeidung wäre die Mischung von Wohn- und Arbeitsstätten dann optimal, wenn ein möglichst ausgeglichenes Verhältnis von Erwerbspersonen und Arbeitsplätzen auf möglichst kleinräumiger Ebene vorliegt. Die Untersuchung für die Nordwestschweiz zeigt, dass sich zwischen 1991 und 1998 das Verhältnis von Erwerbspersonen und Arbeitsplätzen auf Ebene der Umlandgemeinden trotz Bevölkerungs- und Arbeitsplatzverlagerung aus der Kernstadt Basel per Saldo kaum verändert hat. Auf Bezirksebene geht die Suburbanisierung mit einem per Saldo ausgewogeneren Verhältnis von Erwerbspersonen und Arbeitsplätzen einher. Diese Zunahme der dezentralen Mischung von Arbeiten und Wohnen in der Fläche bietet Chancen für eine Reduzierung der Arbeitswege. Sie stellt jedoch für den öffentlichen Verkehr eine Herausforderung dar, wenn die bisher überwiegend auf Basel gerichteten Verkehrsströme zunehmend einem kleinräumigeren Wegemuster weichen sollen.

\section{Summary: Can Population Growth and Increased} Employment Opportunities in the Suburbs Lead to a Decentralised Concentration of Working and Living? An empirical investigation based on North-West Switzerland

In North-West Switzerland, the spatial concentration of population growth and employment opportunity is shifting away from the agglomeration centre of Basel to the surrounding area. The small communities generally profit more from this process than the regional subcentres. The suburbanisation apparent in the agglomeration has also affected the spatial distribution and 
concentration of housing and work opportunities. From the perspective of traffic planning, a balance of work supply and demand within a limited area would be optimal. This investigation of suburbanisation processes within North-West Switzerland shows that despite increasing urban flight/movement away from the urban centres, at the level of the communities the relation between total employed persons and total number of work-places hardly changed between 1991 and 1998. At the district level, a slight improvement in this respect was registered. The increasingly decentralised mix of working and living opens opportunities to reduce travel distance to work. This, however, faces public transport with the challenge of re-orientation: from predominantly feeding the city centre towards catering for local destination demands.

Résumé: Croissance suburbaine de la population et de l'emploi, une voie vers une combinaison décentralisée de l'habitat et du travail? - Une étude empirique à l'aide de l'exemple du nord-ouest de la Suisse

Dans le nord-ouest de la Suisse, les centres spatiaux de la croissance de la population et de l'emploi quittent le cœur de l'agglomération bâloise pour ses environs. $\mathrm{Ce}$ ne sont pas les centres régionaux secondaires qui en profitent le plus, mais surtout les petites communes. Ces processus de suburbanisation se répercutent également sur l'attribution spatiale des lieux de résidence et de travail. Du point de vue de la réduction du trafic, l'attribution des lieux de résidence et de travail serait optimale si le rapport entre les personnes actives et les emplois était le plus équilibré possible, à la plus petite échelle possible. L'étude menée au nord-ouest de la Suisse indique qu'entre 1991 et 1998 le rapport entre les personnes actives et les emplois a peu changé au niveau communal, malgré la désurbanisation croissante de l'habitat et du travail. Au niveau des districts, la suburbanisation s'accompagne même d'un rapport plus équilibré entre les personnes actives et les postes de travail. Cette augmentation de la combinaison décentralisée du travail et de l'habitat devrait permettre de réduire les trajets jusqu'au lieu de travail. Elle représente cependant un défi pour les transports publics: les flux de trafic, jusqu'ici principalement orientés sur Bâle, devront de plus en plus faire place à un modèle de déplacement à plus petite échelle, mais diffus.

Dipl.-Volksw., Lic.rer.reg. Rainer Volman, Geographisches Institut der Universität Basel, Abteilung Humangeographie / Stadt- und Regionalforschung, Klingelbergstr. 16, CH-4056 Basel.

e-mail: rainer.volman@unibas.ch 